\title{
Geometric Correction for Thermographic Images of Asteroid 162173 Ryugu by TIR (Thermal Infrared Imager) onboard Hayabusa2
}

Takehiko Arai ( $\square$ arai.takehiko@ashitech.ac.jp )

Ashikaga Daigaku https://orcid.org/0000-0002-5711-7731

Tatsuaki Okada

Japan Aerospace Exploration Agency

Satoshi Tanaka

Japan Aerospace Exploration Agency

Tetsuya Fukuhara

Rikkyo University

\section{Hirohide Demura}

The University of Aizu

\section{Toru Kouyama}

National Institute of Advanced Industrial Science and Technology

Naoya Sakatani

Rikkyo University

Yuri Shimaki

Japan Aerospace Exploration Agency

Hiroki Senshu

Chiba Institute of Technology

Tomohiko Sekiguchi

Hokkaido University of Education

Jun Takita

Hokkaido Kitami Hokuto High School

Naru Hirata

The University of Aizu

Yukio Yamamoto

Japan Aerospace Exploration Agency

Full paper

Keywords: Hayabysa2, Asteroid, Ryugu, Thermal Infrared Imager, TIR, Shape model, Thermal model, Surface roughness 
Posted Date: January 25th, 2021

DOI: https://doi.org/10.21203/rs.3.rs-48074/v2

License: (c) (i) This work is licensed under a Creative Commons Attribution 4.0 International License. Read Full License 


\section{Geometric Correction for Thermographic Images of Aster-}

\section{oid 162173 Ryugu by TIR (Thermal Infrared Imager) onboard \\ Hayabusa2}

4 Takehiko Arai, Division of Systems and Information Engineering, Ashikaga University,

Ashikaga 326-8558, Japan, arai.takehiko@ashitech.ac.jp

Tatsuaki Okada, Japan Aerospace Exploration Agency, okada@planeta.sci.isas.jaxa.jp

Satoshi Tanaka, Japan Aerospace Exploration Agency, tanaka@paneta.sci.isas.jaxa.jp

Tetsuya Fukuhara, Rikkyo University, tetsuyaf@rikkyo.ac.jp

Hirohide Demura, The University of Aizu, demura@u-aizu.ac.jp

Toru Kouyama, National Institute of Advanced Industrial Science and Technology,

t.kouyama@aist.go.jp

Naoya Sakatani, Rikkyo University, sakatani@rikkyo.ac.jp

Yuri Shimaki, Japan Aerospace Exploration Agency, shimaki@planeta.sci.isas.jaxa.jp

Hiroki Senshu, Chiba Institute of Technology, senshu@perc.it-chiba.ac.jp

Tomohiko Hekiguchi, Hokkaido University of Education,

sekiguchi.tomohiko@a.hokkyodai.ac.jp

Jun Takita, Hokkaido Kitami Hokuto High School, takita@planeta.sci.isas.jaxa.jp

Naru Hirata, The University of Aizu, naru@u-aizu.ac.jp

Yukio Yamamoto, Japan Aerospace Exploration Agency, yukio@planeta.sci.isas.jaxa.jp 


\section{Abstract}

7 The thermal infrared imager (TIR) onboard the Hayabusa2 spacecraft performed thermographic

8 observations of the asteroid 162173 Ryugu (1999 $\mathrm{JU}_{3}$ ) from June 2018 to November 2019. Our previous 9 reports showed the surface temperature and thermal inertia maps of Ryugu. At that time, the mapping 10 procedure from observed TIR images to the shape model of Ryugu has been developed as a geometric 11 correction method. The method used the shape model derived from the observed images by the optical 12 navigation camera (ONC), a numerical geometry toolkit, SPICE kernels (NASA/NAIF) based on the 13 spacecraft attitude data derived from ONC images, and the measured altitudes by the light detection 14 and ranging (LIDAR). The pointing directions of TIR were calculated using an interpolation of data 15 from SPICE kernels during the periods when the ONC or LIDAR observation was performed. Still, the 16 mapping accuracy of the observed TIR images was degraded when the ONC and LIDAR observations ${ }_{17}$ were not performed with TIR. In this paper, to solve the problem of the mapping procedure, we 18 improved the correction method by matching the TIR images, which carried out a one-to-one 19 correspondence between the observed points in the TIR images and the surface nodes addressed on the 20 shape model of Ryugu. This geometric correction adjusted the pointing direction of TIR by rotating ${ }_{21}$ the TIR frame relative to the base of the Hayabusa2 frame using a least squares fit. The resulting 22 temperature maps spatially spreading more than $10^{\circ}$ were improved after the correction, and ${ }_{23}$ high-resolved $0.5^{\circ}$ by $0.5^{\circ}$ maps were constructed. The geometric correction was effective for ${ }_{24}$ characteristic regions where the terrain was comparable to the pixel scale of TIR, such as the Ejima

25 Saxum. The estimated thermal inertia of the bottom of Ejima Saxum was approximately 300

${ }_{26} \mathrm{Jm}^{-2} \mathrm{~S}^{-0.5} \mathrm{~K}^{-1}$. Thus this estimation was succeeded in case that the surface topographic features were

${ }_{27}$ the pixel scale of TIR. However, the thermal inertia estimation of smooth terrains, such as the

${ }_{28}$ Urashima crater, was difficult because of surface roughness effects, where roughness was probably less 29 than the pixel scale of TIR.

\section{Keywords}

31 Hayabysa2, Asteroid, Ryugu, Thermal Infrared Imager, TIR, Shape model, Thermal model, Surface 32 roughness. 
34 Hayabusa2 is a Japanese asteroid sample return mission that rendezvoused with the asteroid 162173

35 Ryugu (previously known as $1999 \mathrm{JU}_{3}$ ) from June 2018 to November 2019 (Tsuda et al. 2013). Ryugu

36 is classified as a C-type asteroid (Binzel et al. 2004), which is considered a parent body of carbonaceous

37 chondrites. Based on the ground and space telescopic observations of Ryugu before the arrival of the

38 Hayabusa2, its rotation period was found to be 7.63 hours, and the thermal inertia was estimated to be 39 $150 \sim 300 \mathrm{Jm}^{-2} \mathrm{~S}^{-0.5} \mathrm{~K}^{-1}$ (Müller et al. 2017).

40 After the arrival of Hayabus2, the observation using the near-infrared spectrometer (NIRS3) suggested

${ }_{41}$ that the surface materials of Ryugu are similar to thermally or shock-metamorphosed carbonaceous

42 chondrites Kitazato et al. (2019). The ONC onboard Hayabusa2 found several characteristic features

${ }_{43}$ of the surface on the scale of centimeters to meters; they are large ridges, regolith deposits, and cracked

44 rocks (Sugita et al. 2019). The gravitational observation indicated that the bulk density of Ryugu is

${ }_{45} 1.19 \pm 0.02 \mathrm{gcm}^{-3}$ (Watanabe et al. 2019). These results implied that Ryugu has a porous, coalesced

46 rubble piles internal structure.

47 The TIR onboard Hayabusa2 is a thermographic camera with $328 \times 248$ pixels resolution (Okada et al.

48 2017). Its sensor is an array of microbolometers, and one pixel has a size of $37 \mu \mathrm{m}$. The observation

49 wavelength is integration energy ranges from 8 to $12 \mu \mathrm{m}$. The field of view is an angle of $16.74^{\circ} \times 12.66^{\circ}$,

50 and the spatial resolution is $0.051^{\circ} /$ pixel. The goal of TIR is to reveal the thermo-physical states of

51 Ryugu's surface, such as grain size and/or its porosity (e.g. Grott et al. 2020), which affects the orbit of

52 the asteroid due to radiation force (Yarkovsky effect, Bottke et al. 2006). The thermo-physical state is

${ }_{53}$ usually characterized by the thermal inertia, which is written as $\Gamma=\sqrt{\rho c_{p} k}$, where $\rho$ is the bulk density,

${ }_{54} k$ is the thermal conductivity, and $c_{p}$ is the specific heat.

${ }_{55}$ The thermal images of Ryugu observed by TIR on 1 August 2018 (the mid-altitude observation, or Mid-

${ }_{56}$ Alt) indicated that the brightness temperature of the surface is broadly homogeneous over the observed

57 hemisphere, which results in flat temperature profiles (Okada et al. 2020; Shimaki et al. 2020). This

${ }_{58}$ phenomenon is considered to be caused by surface roughness within a spatial resolution of the sensor

59 (e.g. Groussin et al. 2007). From the results of the Mid-Alt, the global thermal inertia of Ryugu was 60 estimated by two thermophysical models, TPM-1 and TPM-2 (Okada et al. 2020; Shimaki et al. 2020).

${ }_{61}$ The TPM-1 utilized a shape model of Ryugu (SFM 200k v20180804, Watanabe et al. 2019), including ${ }_{62}$ the secondary radiations and solar ray scatterings from surrounding polygons. It treats a roughness effect 
63 as a reduction of the effective emissivity (Takita et al. 2017). TPM-1 could not reproduce the observed 64 temperature distribution of Ryugu because the effect of surface roughness on the apparent brightness 65 temperatures was not implemented. Still, the TPM-1 reproduced the hot region at the bottom of the ${ }_{66}$ large boulder well (e.g., Catafo Saxum in Figure 2 in Okada et al. 2020). By comparing the observed ${ }_{67}$ data and TPM-1 on the Mid-Alt, the global thermal inertia of Ryugu was roughly estimated to be 300 ${ }_{68} \pm 100 \mathrm{Jm}^{-2} \mathrm{~s}^{-0.5} \mathrm{~K}^{-1}$ (Okada et al. 2020).

69 By contrast, TPM-2 utilized a local shape model of rough surface to successfully reproduce the observed 70 temperature profiles of Ryugu (Shimaki et al. 2020). TPM-2 simulated the solar incident and thermal 71 emission of a local rough surface, which corresponds to a region smaller than a spatial resolution of TIR. 72 With TPM-2, we obtained the global thermal inertia and surface roughness maps of Ryugu; the mean 73 value was estimated to be $225 \pm 45 \mathrm{Jm}^{-2} \mathrm{~S}^{-0.5} \mathrm{~K}^{-1}$ with the mean RMS slope of $47 \pm 5^{\circ}$ (Shimaki et 74 al. 2020). Still, TPM-2 does not utilize the shape model of Ryugu; it cannot reproduce the observed 75 temperature where the topography changes drastically, such as large boulders (Ejima Saxum, Figure 12 76 in Shimaki et al. 2020).

77 Besides the limitation of TPM-2, the accuracy of the TIR image-mapping onto the shape model of 78 Ryugu affects the quality of the observed temperature profiles. The projection was carried out using 79 the numerical geometry tool kit, SPICE kernels (NASA/NAIF). It includes Ryugu's shape models, the 80 TIR alignment, spacecraft attitudes, and spacecraft altitudes. The attitudes and altitudes are based on 81 Hayabusa2 SPICE kernels derived from ONC images and measured altitude by LIDAR. The pointing ${ }_{82}$ directions of TIR were calculated using an interpolation of data from SPICE kernels during the periods 83 when the ONC or LIDAR observation was performed. Still, the mapping accuracy of the observed ${ }_{84}$ TIR images was degraded when the ONC and LIDAR observations were not performed with the TIR 85 observations.

${ }_{86}$ The TIR alignment is defined by the TIR frame relative to the Hayabusa2 frame as Euler angles. The ${ }_{87}$ resulting value of the Euler angles was firstly determined in the Moon observation (Moon distance $\left.{ }_{88}>763,000 \mathrm{~km}\right)$ during the Earth swing-by, $\left(\theta_{z}, \theta_{y}, \theta_{x}\right)=(-1.077,-180.080,0.141)$ (hyb2_v09.tf, SPICE ${ }_{89}$ kernel). These Euler angles were a specific value for TIR as a $2 \mathrm{D}$ imager because the observed target was 90 a point source. We updated the Euler angles just after the arrival of Hayabusa2 using the observation 91 data of 2018-08-01 (Mid-Alt) (Okada et al. 2020; Shimaki et al. 2020), and the resulting value ${ }_{92}$ was $\left(\theta_{z}, \theta_{y}, \theta_{x}\right)=(-1.052,-180.007,0.115)$ (hyb2_v14.tf, SPICE kernel). These Euler angles were the 
mean value determined by using the same method of geometric correction in this study. Still, we found that the $\theta_{z}$, the rotation of the TIR boresight, has a bimodal path in the clockwise and the counterclockwise rotations. This value was also specific and inadequate for other observation data.

Therefore, we recalculated the Euler angles using data from other Ryugu observations in this study.

In this paper, to solve the problem above, we developed a geometric correction by matching the TIR images, which carried out a one-to-one correspondence between the observed points in the TIR images and the surface nodes addressed on the shape model of Ryugu. We show the corrected projection maps and discuss the temperature and thermal inertia estimations, which depended on the topographic variation of the Ryugu surface, resulting in the geometric correction.

\section{Data}

We adapted a geometric correction for all images observed in the early major observations period (Box-A on 2018-07-10, Box-C on 2018-07-20, Mid-Alt on 2018-08-01, and Box-B on 2018-08-31). Using a shape model of Ryugu was 786,432 nodes (SFM 800k v20180804) derived from ONC observations, which was less than the pixel resolution of TIR.

\section{TIR Images}

TIR took Ryugu's thermal images as brightness temperature, which is represented as thermal emission from a black body in radiative equilibrium with sunlight (e.g. Hapke 2012). The nominal observations of Hayabusa2 were called Box-A, -B, and -C (Figure S1 Watanabe et al. 2019). The Box-A (Home Position) was performed at an altitude of around $20 \mathrm{~km}$ at the sub-Earth point. The Box-B was performed at an altitude of $20 \mathrm{~km}$, moving the horizontal direction within $9 \mathrm{~km}$. The Box-C was performed at a low altitude below $20 \mathrm{~km}$ at the sub-Earth point. Besides the Box observations, several observations were carried out at low altitudes, such as the Mid-Altitude operation at an altitude of $5 \mathrm{~km}$ and the MASCOT deployment operation at an altitude of about $50 \mathrm{~m}$ (Jaumann et al. 2019).

The observed data of TIR were converted from digital data to brightness temperature using a calibration database "HEAT" (Endo et al. 2017). The calibration table was constructed in prelaunch experiments (Okada et al. 2020; Arai et al. 2017). The observed data are released in JAXA/DARTS (Yamamoto et al. 2016) and NASA/PDS, which are raw digital images (Level 1 product) and brightness temperature images (Level 2 product). 


\section{Shape model of Ryugu}

The surface terrain of Ryugu has been modeled by numerical shape models constructed from ONC images (Watanabe et al. 2019). The shape models were derived by a structure from motion (SfM) method (e.g. Szeliski 2010) and a stereo photoclinometry (SPC) method (Gaskell et al. 2010). The 3M, 800k, and 200k shape models of Ryugu are composed of 3,145,728, 786,432, and 196,608 triangles, and the sizes of the triangles are tens centimeters, about 1 meter, and a few meters, respectively. We used the SFM (800k) model in this study because the SPICE kernel for the SFM model was compatible with TIR analysis.

\section{SPICE kernels}

NASA's numerical toolkit for the geometry system of the space mission called SPICE kernels (NASA/NAIF) for the Hayabusa2 mission has been released on the website of JAXA/DARTS. The SPICE kernels include the spacecraft information on trajectories derived from data of the orbit control system (AOCS) and LIDAR. The observation coordinate of TIR could be calculated by complementing data of ONC and LIDAR observation when TIR and ONC nominally took images alternately. Still, the pointing coordinate of TIR was uncertain when observations with ONC and LIDAR were not performed. The alignment information of TIR is included in a frame kernel (FK) of the SPICE kernel.

We analyzed data of early major observations (Box-A on 2018-07-10, Box-C on 2018-07-20, Mid-Alt on 2018-08-01, and Box-B on 2018-08-31) using version 03 of the SPICE kernel, released in March 2020. Version 03 kernels contain the improved trajectory of Hayabusa2 derived from LIDAR (Matsumoto et al. 2020) but do not include the relative position and attitude between Ryugu and Hayabusa2 derived from the shape model. The new version of the SPICE kernels for Hayabusa2 will be released in 2021 and includes a precise attitude of Hayabusa2 derived from the shape model, along with the results of this study.

\section{Method: Geometric Correction}

To fit positions observed by TIR to the coordinates of a shape model, performed by rotating the TIR image frame using the SPICE toolkit (N0066), are geometric corrections for the TIR brightness temperature images. The temperatures of the observation images are represented by $T(i, j)$ in the image pixel coordinate, where $i$ and $j$ are the index of pixels $(i=1 \sim 328, j=1 \sim 248)$, respectively. On the other hand, the observed brightness temperatures projected on facets of a shape model are represented by $T(X, Y, Z)$ in the Cartesian coordinate of the Ryugu system (Figure 1), where the positional vector 

176 is written as the sum of the polygon areas, as follows:

$$
S=\sum_{k} s_{k} \cos \phi_{k}
$$

\section{Projection from Image to Shape}

\section{Projection from Shape to Image}

The brightness temperature projection from a TIR image to a shape model of Ryugu is expressed as a coordinate transformation from the pixel coordinate to the Ryugu coordinate. We assume that the observed brightness temperature of a pixel value is the mean temperature of the observed region because the TIR pixel detects total radiation fluxes from a region of the Ryugu surface (e.g., $\sim 5 \mathrm{~m}$ squares in Mid-Alt). The brightness temperature value of an image is directly converted to a shape model as $T(i, j) \rightarrow T(X, Y, Z)$. This transformation is represented by using the vector sum of positional vectors. A ray vector $\mathbf{V}_{R}$ from a polygon of the shape model of Ryugu to TIR is written as $\mathbf{V}_{R}=\mathbf{V}_{T}-\mathbf{V}_{P}$, where $\mathbf{V}_{\mathrm{P}}$ and $\mathbf{V}_{\mathrm{T}}$ are position vectors of the polygon and TIR, respectively (Figure 1). The ray vector in the coordinated system of Ryugu $\mathbf{V}_{\mathrm{R}}$ is transformed into that of the coordinate system of Hayabusa2 $\mathbf{v}_{\mathrm{R}}$ using a coordinate conversion tool of the SPICE kernels, as $\mathbf{V}_{\mathrm{R}} \rightarrow \mathbf{v}_{\mathrm{R}}$. A normalized boresight vector of TIR in the coordinated system of TIR is $(x, y, z)=(0,0,1)$. The frame of TIR is the coordinate system of Hayabusa2 rotated $-180^{\circ}$ in the y-axis (Figure 1). The focal point length of the TIR is $42.2 \mathrm{~mm}$, and thus the positional vector of the focal point is written as $\mathbf{v}_{\mathrm{F}}(x, y, z)=(0,0,0.0422)$. The incident rays from polygons of the shape model to TIR intersect the image plane of TIR at the positional vector $\mathbf{v}_{\mathrm{I}}$, where $\mathbf{v}_{\mathrm{I}}=\mathbf{v}_{\mathrm{R}}+\mathbf{v}_{\mathrm{F}}$. The pixel size of the TIR sensor is 37 square $\mu \mathrm{m}$, and thus the scalar value $\left|\mathbf{v}_{\mathbf{I}}(x, y, z)\right|$ normalized by the pixel size is converted to the pixel coordinate $(i, j)$.

The projection temperature from a shape to an image is written as an inverse process of the image projection to shape coordinate. Still, this reprojection process is irreversible because it is a not one-to-

$$
F=\frac{1}{\pi} \sum_{k} \epsilon_{k} \sigma T_{k}^{4} s_{k} \cos \phi_{k}+\text { scattering, }
$$

where $\epsilon_{k}$ is the emissivity, $\sigma$ is the Boltzmann constant, $s_{k}$ is the area of the polygons, $\phi_{k}$ is the emission angle to the TIR direction, and $k$ denotes the index of the polygon. The observation area for one pixel 

197 ideally zero. follows:

\section{Fitting Alignment}

The TIR frame $\mathbf{v}_{\mathrm{T}}(x, y, z)$ is defined based on the Hayabusa2 frame $\mathbf{v}_{\mathrm{H}}\left(x_{o}, y_{o}, z_{o}\right)$. The frame transformation is described using the Euler rotation, as follows:

$$
\mathbf{v}_{\mathrm{H}}=\mathrm{R}_{z} \mathrm{R}_{y} \mathrm{R}_{x} \mathbf{v}_{\mathrm{T}},
$$

where $\mathrm{R}_{z}, \mathrm{R}_{y}$, and $\mathrm{R}_{x}$ form the Euler rotation matrix in the right-handed coordinate system. The matrix $\mathrm{R}_{z}$ is the rotation of the TIR boresight vector, which has a bimodal path in the clockwise and the counterclockwise rotations. In this study, $\mathrm{R}_{z}$ is fixed as a unit matrix because this parameter is not effective in minimizing the converge value of the least squares fittings. Hence, Equation (4) is written, as

$$
\left[\begin{array}{l}
x_{o} \\
y_{o} \\
z_{o}
\end{array}\right]=\left[\begin{array}{ccc}
\cos \theta_{y} & \sin \theta_{y} \cos \theta_{x} & -\sin \theta_{y} \sin \theta_{x} \\
0 & \sin \theta_{x} & \cos \theta_{x} \\
-\sin \theta_{y} & \cos \theta_{y} \cos \theta_{x} & -\cos \theta_{y} \sin \theta_{x}
\end{array}\right]\left[\begin{array}{l}
x \\
y \\
z
\end{array}\right],
$$

where $\theta_{x}$ and $\theta_{y}$ are the Euler angles.

TIR observation points are determined by fitting the observation image to the projection image and onto the shape model of Ryugu. This adjustment is performed by minimizing the difference between the observed temperature $T(i, j)$ and the reprojected the Ryugu shape temperature $T^{\prime}(i, j)$ in the image pixel coordinate using the least squares fit, as follows:

$$
\mathrm{RSS}=\sum_{i, j}\left[T(i, j)-T^{\prime}(i, j)\right]^{2},
$$

where RSS is a residual sum of squares in temperature. The free parameters of this fitting are the Euler angles of $\theta_{y}$ and $\theta_{x}$ in Equation (5). The fitting is performed by using the algorithms of the LevenbergMarquardt and Simplex fitting method (e.g. Press et al. 2007). The fitting converged value of RSS is 


\section{Results}

The geometric correction was performed for all observed TIR images in the early major observations, and the observed temperature was projected onto the shape model and geographic maps.

Figure 2 shows an example of the geometric correction flow for an image (Mid-Alt 23:07), which is the observed image projected onto the shape model and reprojected onto images. The best-fit Euler angles as free parameters of $\theta_{y}$ and $\theta_{x}$ were determined by adjusting the observed image and the reproduced image by comparing the residuals of both images. Although the overhang of the projection was at most 7 pixels or about $35 \mathrm{~m}$ (upper row of Figure 2) before the correction, the geometric correction achieved the projection accuracy within one pixel after the correction (bottom row of Figure 2). The observed temperature at the asteroid limb position was not fitted because the pixel value at the limb included not only Ryugu temperatures but also space background.

Table 1 shows the best-fit Euler angles, along with the observation configuration for each observation, such as the solar distance, the solar phase angle, the sub-spacecraft latitude, the spacecraft altitude, and the number of observed images. The error of the Euler angles is the standard deviation of each best-fit value. The resulting value of Box-A was different from the other resulting values of Box-B, C, and Mid-Alt, due to the uncorrected attitude of the spacecraft derived from this version's SPICE kernel. The resulting value of the Box-C and Mid-Alt observations at the low altitude of $5 \sim 7 \mathrm{~km}$ is reliable because orbit and altitude data of the spacecraft was corrected observing with ONC and LIDAR during this period. We believe that this result represents the accurate alignment of TIR determined by the geometric correction. Therefore, the geometric correction achieves the accuracy between the results of $(0,-179.95,0.06)$ and $(0,-179.93,0.09)$. These differences are less than a pixel resolution of TIR $(0.051$ degrees/pixel). We will update the alignment of TIR (FK of SPICE kernel) using these mean values. Figure 3 shows the maximum temperature maps projected onto geographic maps with $0.5^{\circ}$ by $0.5^{\circ}$ gridded. These maximum temperatures were merged and extracted, combined with all observed images of the Ryugu's one rotation period obtained with the observed time interval of 76 seconds before and after the correction (Box-A, Box-C, Mid-Alt, and Box-B). We can see that the temperature maps spatially spreading more than $10^{\circ}$ are improved after the correction, where the spreading areas are converged in 5 the size of $0.5^{\circ}$ gridded (See the Ejima Saxum and Otohime Saxum). The geometric correction succeeded 226 in detailed map construction with the high resolution of $0.5^{\circ}$ gridded for Box-C and Mid-Alt because of ${ }_{227}$ low altitude observations. The geometric correction is also effective for observed data at a high altitude 


\begin{tabular}{cccccccc}
\hline Observation & Sun Distance & Phase Angle & SC Latitude & Altitude & Image & Euler $\bar{\theta}_{Y}$ & Euler $\bar{\theta}_{X}$ \\
- & $(\mathrm{AU})$ & $($ degree $)$ & $($ degree $)$ & $(\mathrm{km})$ & - & $($ degree $)$ & $($ degree $)$ \\
\hline Box-A & 1.005 & 18.57 & -4.33 & 19.58 & 64 & -179.78 & 0.08 \\
$(2018-07-10)$ & - & \pm 0.07 & \pm 0.01 & \pm 0.05 & - & \pm 0.13 & \pm 0.01 \\
\hline Box-C & 1.028 & 18.45 & -4.93 & 6.59 & 96 & -179.95 & 0.06 \\
$(2018-07-20)$ & - & \pm 0.12 & \pm 0.08 & \pm 0.08 & - & \pm 0.01 & \pm 0.01 \\
\hline Mid-Alt & 1.060 & 18.98 & -5.37 & 5.28 & 120 & -179.93 & 0.09 \\
$(2018-08-01)$ & - & \pm 0.17 & \pm 0.08 & \pm 0.33 & - & \pm 0.13 & \pm 0.07 \\
\hline Box-B & 1.148 & 40.10 & -1.66 & 21.41 & 96 & -180.00 & 0.08 \\
$(2018-08-31)$ & - & \pm 0.10 & \pm 0.01 & \pm 0.04 & - & \pm 0.01 & \pm 0.01 \\
\hline \hline
\end{tabular}

Table 1. Observation configuration and mean values of the best-fit Euler angles.

observation of $20 \mathrm{~km}$ (Box-A and Box-B) because the temperature distribution was more improved.

\section{Discussions}

The geometric correction carried out the projection within the accuracy of the pixel scale of TIR. The spatially spreading temperature areas were converged in the size of $0.5^{\circ}$ gridded after the correction. Here, we discuss the reproducibility of the observed temperature onto the shape model and map compared with numerical simulation results, such as the specific region of Ejima Saxum and Urashima Crater.

Figure 4 shows examples of the temperature profile in time series, derived from the temperature map projected onto a geographic map using 31 observed images with $0.5^{\circ}$ by $0.5^{\circ}$ gridded. These temperature profiles show the hot area of the Ejima Saxum and Urashima crater. The temperature before the correction showed that of the neighboring area $0.5^{\circ}$ gridded apart after the correction (middle figures of Figure 4). The temperature profile varied at the Ejima Saxum as a characteristic area before and after the correction, but the profile was not significantly changed at the Urashima crater. Thus, the geometric correction is important for the detailed estimation of the temperature profile in topographically characteristic areas.

The observed temperature profiles of the bottom of Ejima Saxum and the Urashima crater and the simulated temperature profiles (TPM-1) using Equation (3) are shown in Figure 4. As a characteristic 24 area with the topographical change, the thermal inertia of the bottom of Ejima Saxum was estimated to 
be $300 \mathrm{Jm}^{-2} \mathrm{~S}^{-0.5} \mathrm{~K}^{-1}$ except the temperature profile of the asteroid limb, including large uncertainty at dawn time $(\sim 14: 30)$ and dusk time $(\sim 17: 00)$. This result indicates that local variation in temperature change should not be effective for Yarkovsky effects even in the characteristic area of Ejima Saxum because the thermal inertia variations are small compared with other area's temperature profiles (Figure 7 Shimaki et al. 2020). On the other hand, the thermal inertia of the Urashima crater was approximately 200 to $400 \mathrm{Jm}^{-2} \mathrm{~S}^{-0.5} \mathrm{~K}^{-1}$ estimated by comparing the simulated profiles. If the thermal inertia at the median passage time (15:48 UTC) represents actual value, the thermal inertia is estimated to be $300 \pm$ $100 \mathrm{Jm}^{-2} \mathrm{~S}^{-0.5} \mathrm{~K}^{-1}$. This result is comparable to (Okada et al. 2020). The variation between the observed profile and the simulated profiles was also probably caused by the surface roughness. These results indicate that the brightness temperatures of observed areas larger than a TIR pixel scale are determined by largescale surface topography that can be reproduced by TPM-1. In contrast, the brightness temperatures of observed areas smaller than a TIR pixel scale are probably determined by small-scale roughness that can be reproduced by TPM-2.

We compared the observed image with the simulated image using TPM-1 mapped onto the shape model, as shown in Figure 5. The hot region is found at the bottom of Ejima Saxum in both of the observed and simulated images (see the closed-up image of ONC, Figure 2(A) Sugita et al. 2019). The simulated parameters were assumed to be an emissivity of 1.0, an albedo of 0.045 (Sugita et al. 2019), and a thermal inertia of $350 \mathrm{Jm}^{-2} \mathrm{~s}^{-0.5} \mathrm{~K}^{-1}$. The observed image (Figure 5a) is similar to the simulated image involving the secondary radiations and the solar ray scatterings (Figure 5b), but not by the simulated image involving only solar ray scatterings (Figure 5c). This result implies that the secondary radiations between surrounding terrains generate the hot region. Although the thermal inertia at the bottom of Ejima Saxum resulted in a thermal inertia profile of approximately $300 \mathrm{Jm}^{-2} \mathrm{~s}^{-0.5} \mathrm{~K}^{-1}$, the simulated images were reproduced well by using the value of $350 \mathrm{Jm}^{-2} \mathrm{~S}^{-0.5} \mathrm{~K}^{-1}$. This difference is probably caused by surface roughness because the surface roughness can reduce the whole temperature of the surface.

The mapped temperature's reproducibility by the geometric correction is satisfactory, but the effects of surface geometry and surface roughness vary the apparent surface temperature. Thus, we conclude that the development of a thermophysical model, including a hybrid model that implements topography and small-scale surface roughness, is required. 


\section{Summaries}

${ }_{274}$ The observed temperature maps - which were projected onto the Ryugu map spatially spreading areas 275 more than $10^{\circ}$ - are improved by the geometric correction developed in this study. The maps were constructed with high-resolved 0.5 by 0.5 gridded. This positioning accuracy of TIR was improved 277 equivalent to a pixel resolution of $5 \mathrm{~m}$ at an altitude of $5 \mathrm{~km}$.

278 The thermal inertia estimation of a characteristic area's observed temperatures was successful when 279 larger than the TIR pixel scale corrected by the surface topography, which the temperature profile can 280 be reproduced by TPM-1 (Takita et al. 2017). In contrast, the thermal inertia estimation of the 281 smooth area's observed brightness temperatures was inaccurate. This was probably caused by surface 282 roughness smaller than the TIR pixel scale, which the temperature profile that can be reproduced by 283 TPM-2 (Shimaki et al. 2020). We conclude that the development of a thermophysical model, including 284 a hybrid model that implements topography and small-scale surface roughness, is required.

285 The alignment value of TIR was determined and is released as the new version FK of the Hayabusa2 ${ }_{286}$ SPICE kernels. The higher degree products of TIR constructed by using the geometric correction are 287 also released on the web site of JAXA/DARTS and NASA/PDS in 2021. 


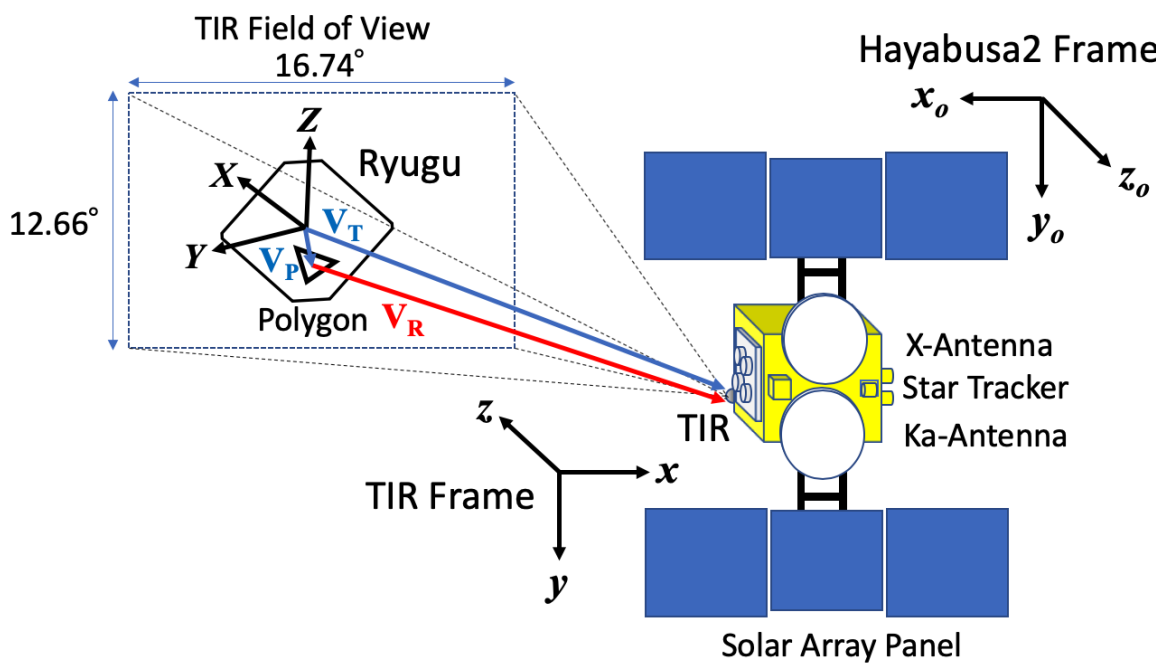

Figure 1. Schematic view of Ryugu coordinate and TIR and Hayabusa2 frames relation. The TIR frame is rotated in the Euler angle of $\theta_{y}=-180^{\circ}$ relative to the Hayabusa2 frame. Rays from a polygon of a Ryugu shape model are incident to TIR. 


\section{Observed Image}

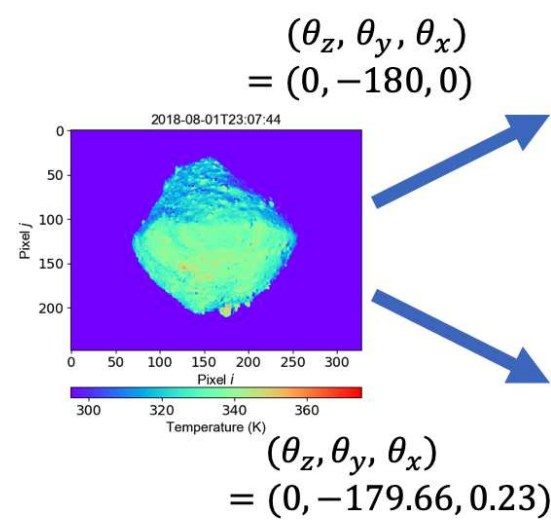

Projected onto Shape
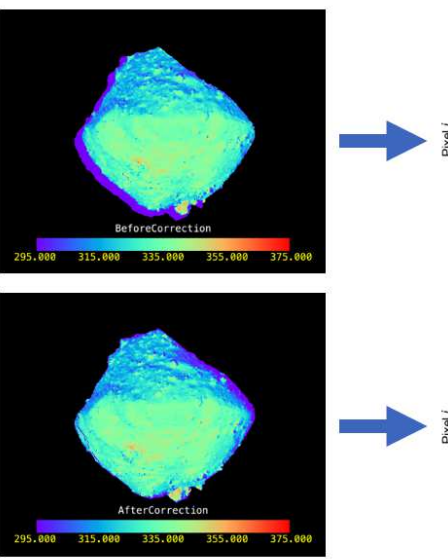

Residual Image
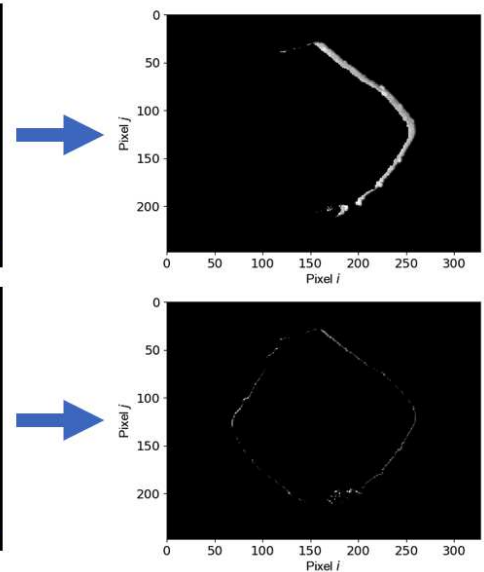

Figure 2. The geometric correction procedure. The figures of upper and bottom rows were generated in the step described in the subsection of Projection from Image to Shape in the method section as follows: "observed image" $\rightarrow$ "projected onto the shape model" $\rightarrow$ "reproduced image." Minimizing the difference between the observed image and the reproduced image derived the best-fit Euler angles $\theta_{y}$ and $\theta_{x}$ using Equation (6), iteratively. The upper row shows the derived figures used by the fitting Euler angles as the initial guess, and the bottom row shows the derived figures fitted using the best-fitted one. The residual of the observed image and the reproduced image (right figures) mean the out of projection, shown in grayscale. 

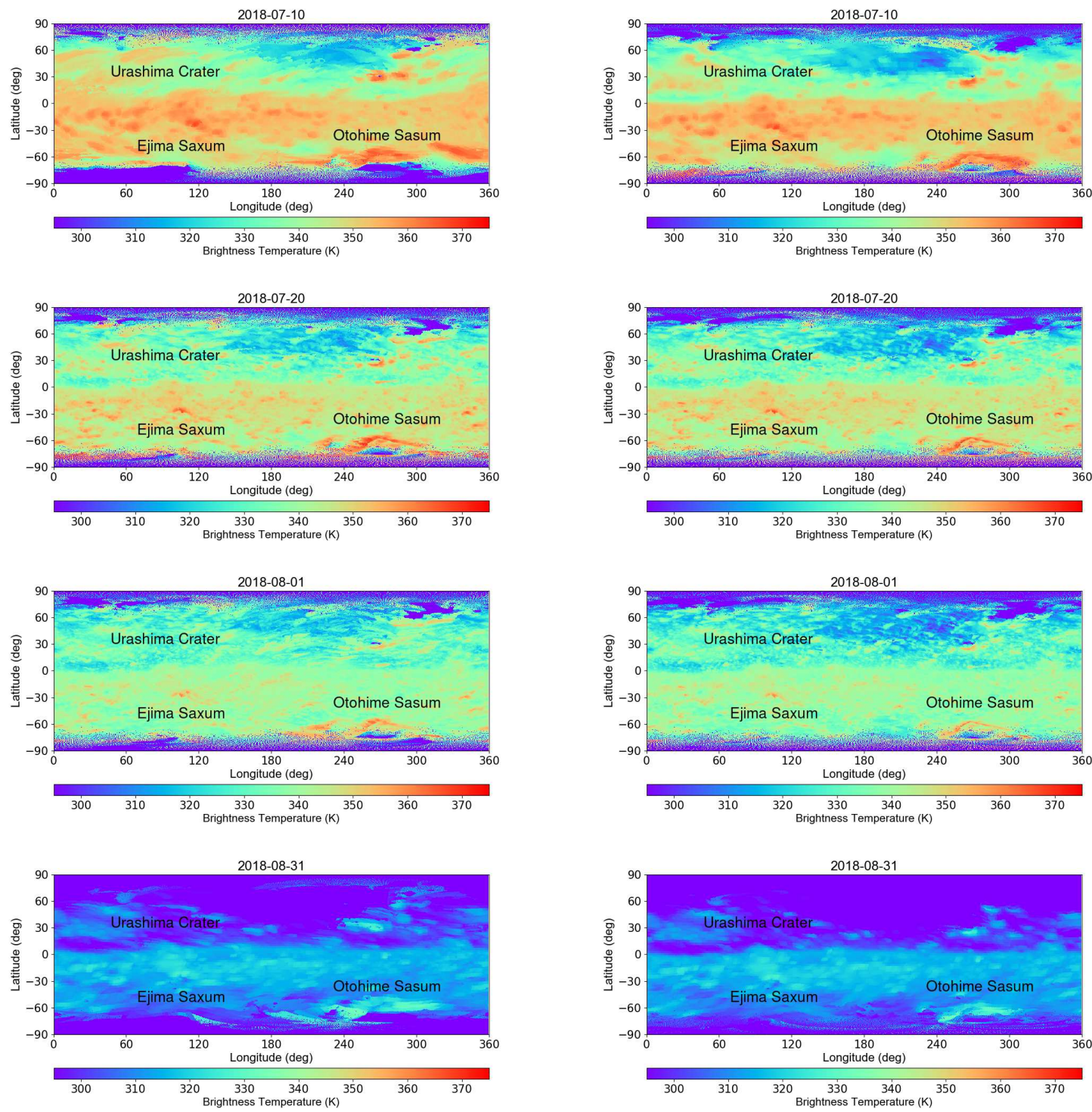

(a) Before correction

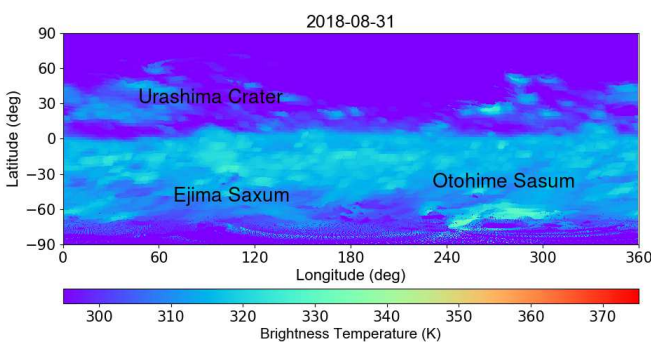

(b) After correction

Figure 3. Maximum temperature projected onto the geographic maps (Box-A, Box-C, Mid-Alt, and Box-B). (a) shows the before-correction map and (b) shows the after-correction map. These maps indicate that observed maximum temperatures merged and extracted combined all observed images for one rotation period of Ryugu projected onto the geographic map with $0.5^{\circ}$ by $0.5^{\circ}$ gridded. 

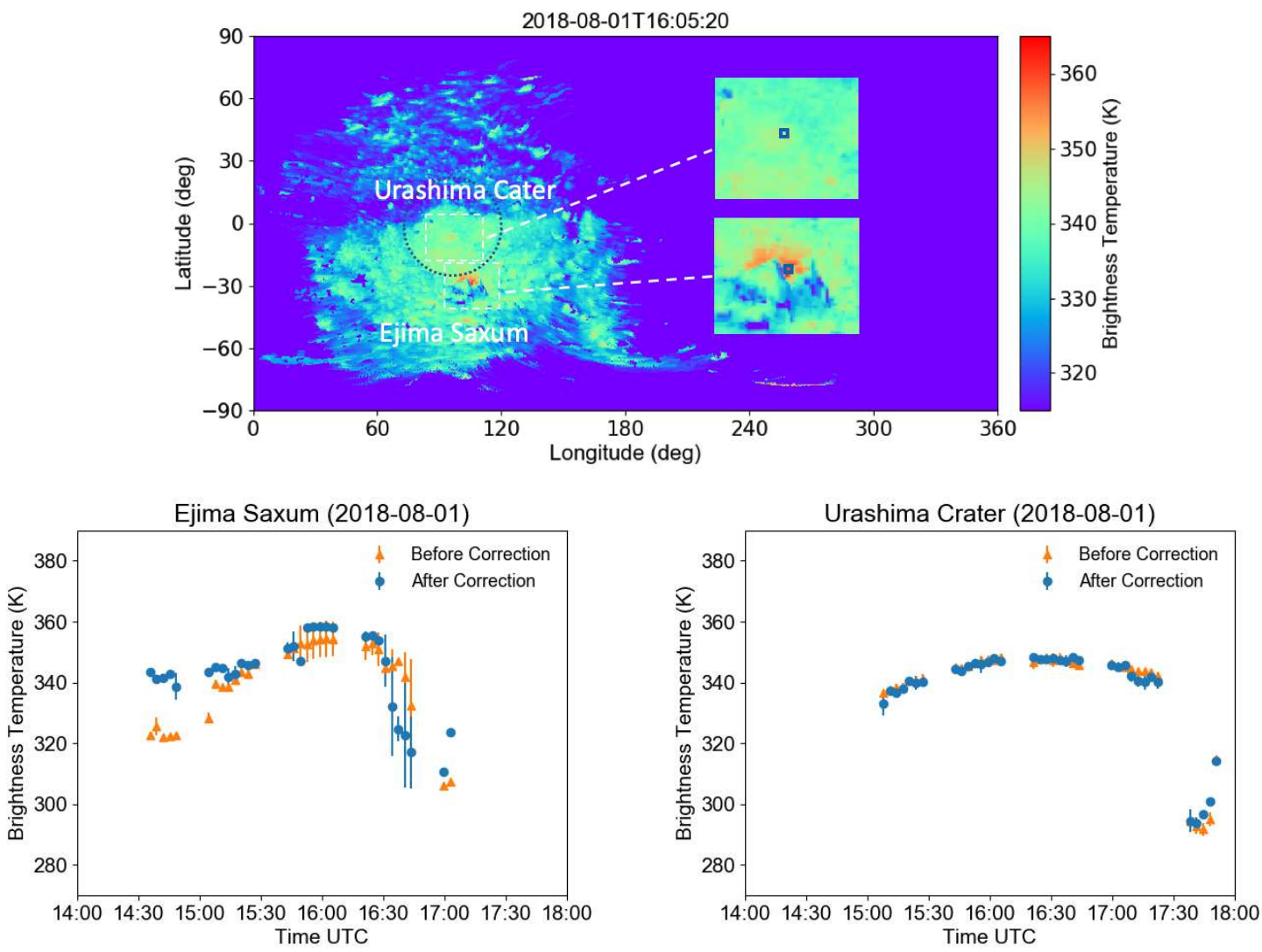

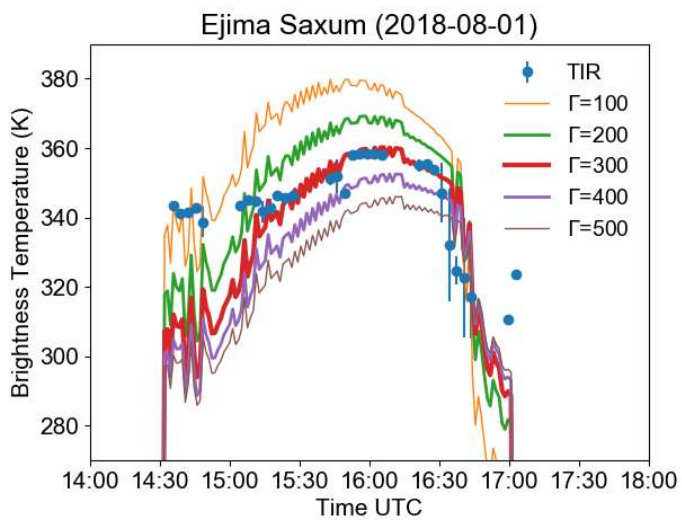

(a) Hot area of Ejima Saxum

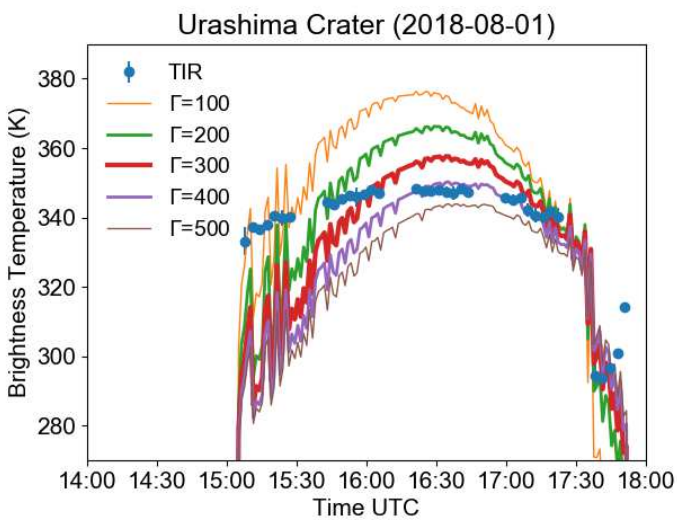

(b) Hot area of Urashima Crater

Figure 4. An example of one projection image (Mid-Alt16:05:20) using the shape model of Ryugu (SFM $800 \mathrm{k}$ v20180804) with $0.5^{\circ}$ by $0.5^{\circ}$ gridded (top) and the temperature profiles (middle and bottom). The temperature profiles were derived from 32 images extracted the hot areas of Ejima Saxum (a; Lat: $-28.0^{\circ}$, Lon: $106.0^{\circ}$ ) and Urashima crater (b; Lat: $-6.5^{\circ}$, Lon: $96.5^{\circ}$ ) as shown in the square area of the top image. These points indicate the observed brightness temperature of TIR by a time interval of 76 seconds, and the error bars are the observed temperature range of the minimum to maximum temperature. These orange points denote temperature before the geometric correction, and blue points denote temperature after geometric correction (middle). Comparison of the temperature profiles after the geometric correction with the simulated temperature profiles, TPM-1 (Takita et al. 2017), is shown 16 (bottom). These lines indicate the simulated thermal inertia $\Gamma$ in steps of $100 \mathrm{Jm}^{-2} \mathrm{~s}^{-0.5} \mathrm{~K}^{-1}$ using the shape model (SFM 200k v20180804). 
289 AOCS: Attitude and Orbit Control System

290

DARTS: Data Archive and Transmission System

291

292

293

294

\section{Declarations}

\section{Availability of data and materials}

${ }_{313}$ The original data of this study are available and downloadable on the following web site:

${ }_{314}$ TIR data: https://www.darts.isas.jaxa.jp/pub/hayabusa2/tir_bundle/browse/

JAXA: Japan Aerospace Exploration Agency

LIDAR: Light Detection and Ranging

MARA: Mobile Asteroid Surface Scout

MASCOT: Mobile Asteroid Surface Scout

Mid-Alt: Middle Altitude

NAIF: Navigation and Ancillary Information Facility

NASA: National Aeronautics and Space Administration

NIRS3: Near Infrared Spectrometer

ONC: Optical Navigation Camera

RCS: Reaction Control System

RSS: Residual Sum of Squares

RW: Reaction Wheel

SC: Spacecraft

SfM: Structure from Motion

SPC: Stereo Photoclinometry

SPICE: Spacecraft Planet Instrument C-matrix Events

TIR: Thermal Infrared Imager

TPM: Thermophysical Model

$315 \quad \mathrm{SPI}$

SPICE kernels: https://www.darts.isas.jaxa.jp/pub/hayabusa2/spice_bundle/document/spiceds_v001.html 
317 The authors declare that they have no competing interests.

\section{$318 \quad$ Funding}

The JSPS Kakenhi JP17H06459 partly supports TO.

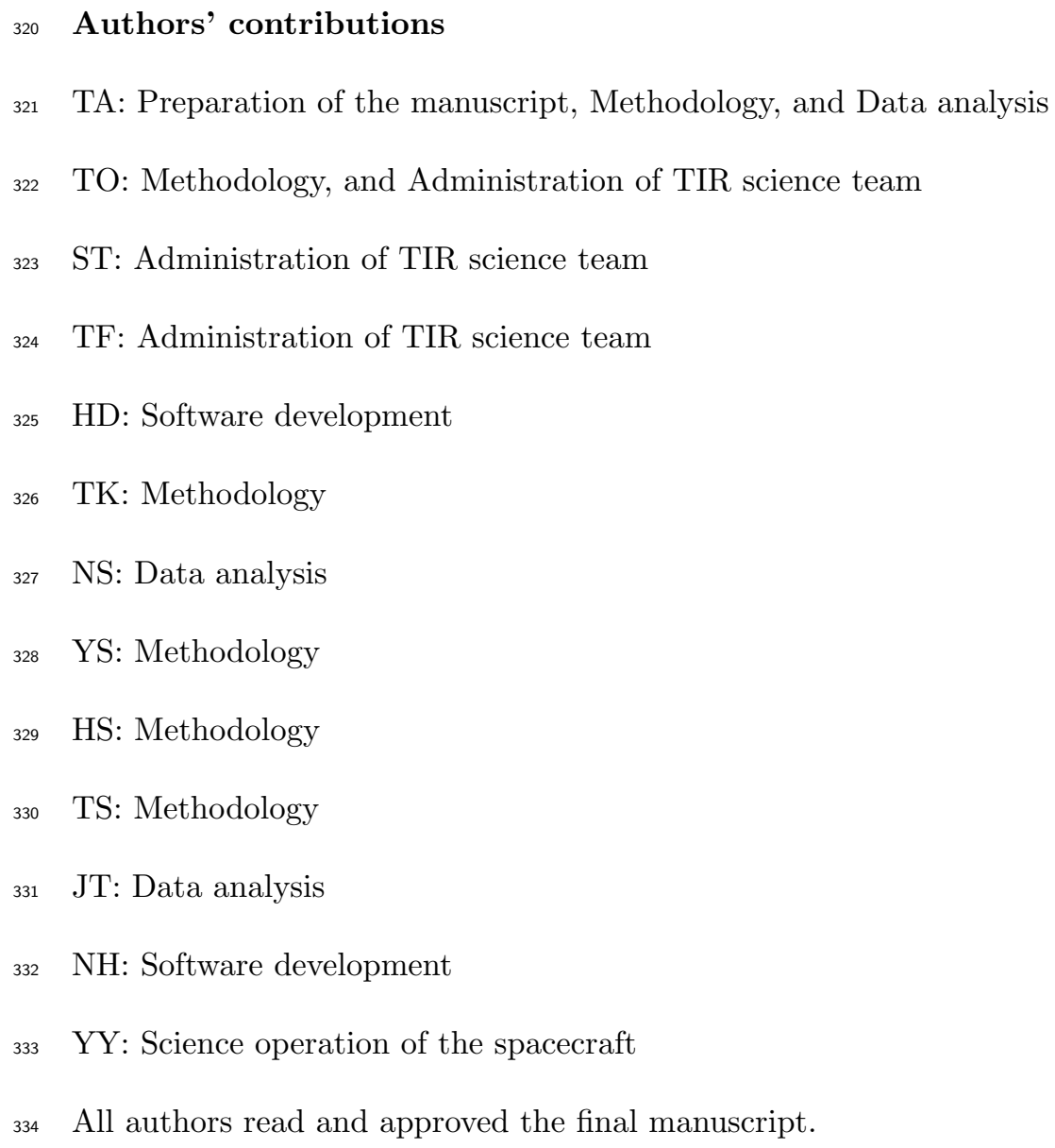

\section{Authors' information}

336 TA: Division of Systems and Information Engineering, Ashikaga University, Ashikaga 326-8558, Japan.

337 TO: Institute of Space and Astronautical Science, Japan Aerospace Exploration Agency (JAXA), Sagami338 hara 252-5210, Japan.

з39 TO: The University of Tokyo, Tokyo 113-0033, Japan

${ }_{340}$ ST: Institute of Space and Astronautical Science, Japan Aerospace Exploration Agency (JAXA), Sagami341 hara 252-5210, Japan.

342 ST: The Graduated University for Advanced Studies, SOKENDAI, Hayama 240-0193, Japan. 
TF: Rikkyo University, Tokyo 171-8501, Japan.

HD: The University of Aizu, Aizu-Wakamatsu 965-8580, Japan.

TK: National Institute of Advanced Industrial Science and Technology (AIST), Tokyo 135-0064, Japan. NS: Rikkyo University, Tokyo 171-8501, Japan.

YS: Institute of Space and Astronautical Science, Japan Aerospace Exploration Agency (JAXA), Sagamihara 252-5210, Japan.

HS: Planetary Exploration Research Center, Chiba Institute of Technology, Narashino 275-0016, Japan.

TS: Hokkaido University of Education, Sapporo 002-8501, Japan.

JT: Hokkaido Kitami Hokuto High School, Kitami 090-0035, Japan.

NH: The University of Aizu, Aizu-Wakamatsu 965-8580, Japan.

YY: Institute of Space and Astronautical Science, Japan Aerospace Exploration Agency (JAXA), Sagamihara 252-5210, Japan.

\section{Acknowledgments}

We are grateful to all members of the Hayabusa2 team and thank NEC Inc. Co., who made the flight model of TIR. We thank Dr. Tsuneo Matsunaga of National Institute for Environmental Studies, who provides us computer resources.

\section{References}

Arai T. et al. (2017) Thermal Imaging Performance of TIR Onboard the Hayabusa2 Spacecraft. Space Science Reviews 208:239-254. https://doi.org/10.1007/s11214-017-0353-9

Binzel R. P. et al. (2004) Observed spectral properties of near-Earth objects: results for population distribution, source regions, and space weathering processes. Icarus 170(2):259-294. https://doi.org/10.1016/j.icarus.2004.04.004

Bottke W. F. Jr. et al. (2006) The Yarkovsky and YORP Effects: Implications for Asteroid Dynamics. Annu. Rev. Earth Planet. Sci. 34:157-91.

Endo K. et al. (2017) HEAT: Image and database browser for the thermal imager on Hayabusa2. IEEE Aerospace Conference 1-10. https://doi.org/10.1109/AERO.2017.7943827

Gaskell R. W. et al. (2010) Characterizing and navigating small bodies with imaging data. Meteoritics 
\& Planetary Science. 43(6):1049-1061. https://doi.org/10.1111/j.1945-5100.2008.tb00692.x

Grott et al. (2020) Macroporosity and Grain Density of Rubble Pile Asteroid (162173) Ryugu. https://doi.org/10.1029/2020JE006519

Groussin O. et al. (2007) Surface temperature of the nucleus of Comet 9P/Tempel 1. Icarus 187(1):16-25. https://doi.org/10.1016/j.icarus.2006.08.030

Hapke (2012) Thermal emission and emittance spectroscopy, Theory of Reflectance and Emittance Spectroscopy. Cambridge University Press, 412-439. https://doi.org/10.1017/CBO9781139025683

Jaumann R. et al. (2019) Images from the surface of asteroid Ryugu show rocks similar to carbonaceous chondrite meteorites. Science 365:817-820. https://doi.org/10.1126/science.aaw8627

Kitazato K. et al. (2019) The surface composition of asteroid 162173 Ryugu from Hayabusa2 near-infrared spectroscopy. Science 364(6437): 272-275. https://doi.org/10.1126/science.aav7432

Okada T. et al. (2017) Thermal Infrared Imaging Experiments of C-Type Asteroid 162173 Ryugu on Hayabusa2. Space Science Reviews 208: 255-286. https://doi.org/10.1007/s11214-016-0286-8

Okada T. et al. (2018) Earth and moon observations by thermal infrared imager on Hayabusa2 and the application to detectability of asteroid 162173 Ryugu. Planetary and Space Science 158:46-52. https://doi.org/10.1016/j.pss.2018.05.007

Okada T. et al. (2020) Highly porous nature of a primitive asteroid revealed by thermal imaging. Nature 579: 518-522. https://doi.org/10.1038/s41586-020-2102-6

Press W. H. et al. (2007) Modeling of Data, Numerical Recipes, 3rd edn., Cambridge University Press, 773-839.

Shimaki Y. et al. (2020) Thermophysical Properties of the Surface of Asteroid 162173 Ryugu: Infrared Observations and Thermal Inertia Mapping. Icarus 348. https://doi.org/doi.org/10.1016/j.icarus.2020.113835

Sugita S. et al. (2019) The geomorphology, color, and thermal properties of Ryugu: Implications for parent-body processes. Science 364(6437). https://doi.org/10.1126/science.aaw0422

Matsumoto K. et al. (2020) Improving Hayabusa2 trajectory by combining LIDAR data and a shape model. Icarus 338. https://doi.org/10.1016/j.icarus.2019.113574

Szeliski R. (2010) Computer Vision: Algorithms and Applications. Springer.

Takita J. et al. (2017) Feasibility and accuracy of thermophysical estimation of asteroid 162173 Ryugu (1999 $\mathrm{JU}_{3}$ ) from the Hayabusa2 thermal infrared imager. Space Science Reviews 208:287-315. 
Tsuda Y. et al. (2013) System design of the Hayabusa 2 - asteroid sample return mission to $1999 \mathrm{JU}_{3}$.

$403 \quad$ Acta Astronaut 91: 356-362.

${ }_{404}$ Müller T. G. et al. (2017), Hayabusa-2 mission target asteroid 162173 Ryugu (1999 JU3): searching for 405 the object's spin-axis orientation. Astron Astrophys 599:A103.

406 https://doi.org/10.1051/0004-6361/201629134

${ }_{407}$ Watanabe S. et al. (2019) Hayabusa2 arrives at the carbonaceous asteroid 162173 Ryugu - a

408 spinning-top-shaped rubble pile. Science 364(6437):268-272. https://doi.org/10.1126/science.aav8032

409 Yamamoto Y. et al. (2016) Scientific Data Archives in Hayabusa2 Mission. Trans. JSASS Aerospace Tech. Japan 14:151-154. https://doi.org/10.2322/tastj.14.Pk_151 


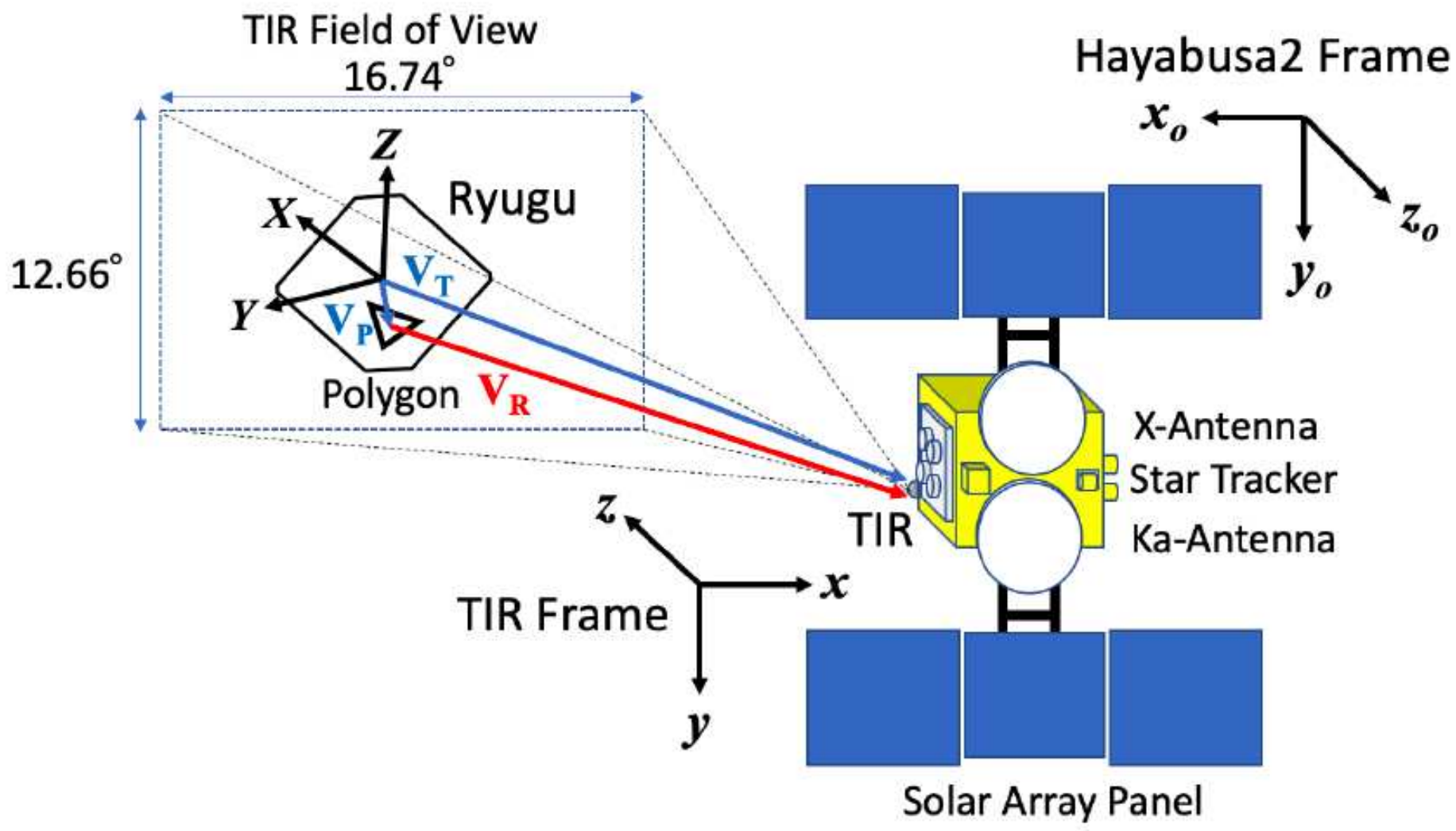

Figure 1

Schematic view of Ryugu coordinate and TIR and Hayabusa2 frames relation. The TIR frame is rotated in the Euler angle of $\theta y=-180^{\circ}$ relative to the Hayabusa2 frame. Rays from a polygon of a Ryugu shape model are incident to TIR. 
Observed Image

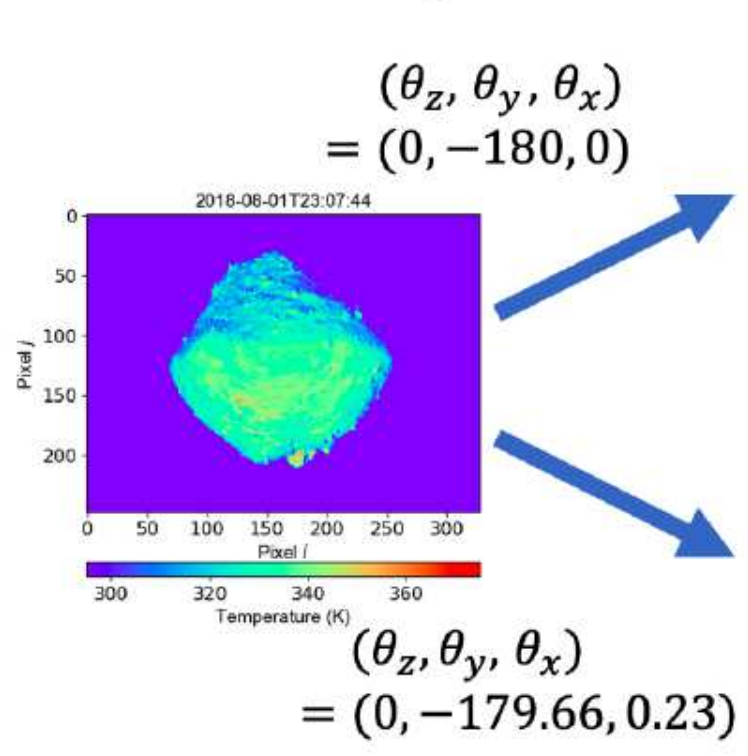

Projected onto Shape
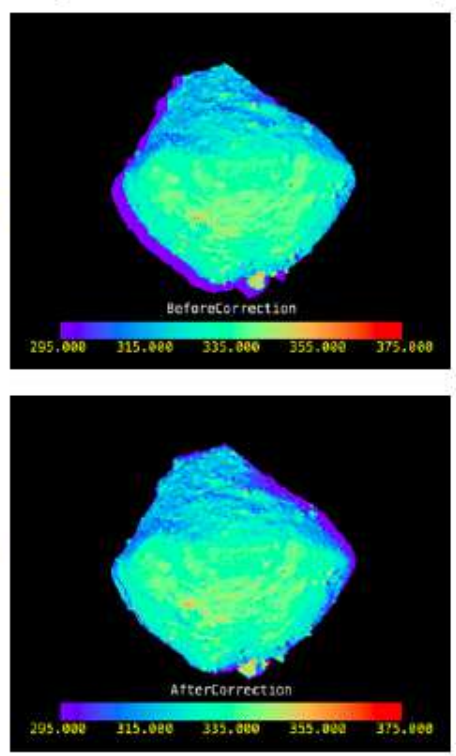

Residual Image
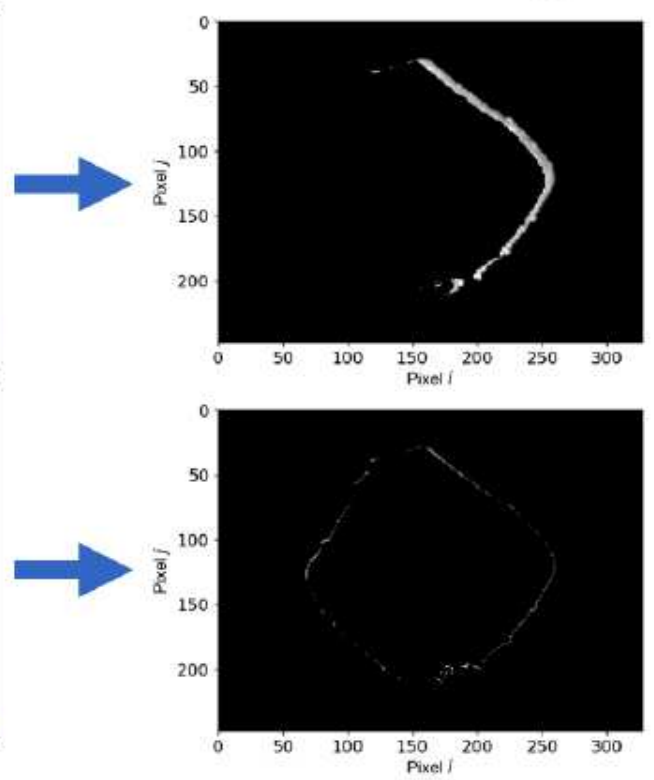

Figure 2

The geometric correction procedure. The figures of upper and bottom rows were generated in the step described in the subsection of Projection from Image to Shape in the method section as follows: "observed image" $\nabla$ "projected onto the shape model" $\nabla$ "reproduced image." Minimizing the difference between the observed image and the reproduced image derived the best-fit Euler angles $\theta y$ and $\theta x$ using Equation (6), iteratively. The upper row shows the derived figures used by the fitting Euler angles as the initial guess, and the bottom row shows the derived figures fitted using the best-fitted one. The residual of the observed image and the reproduced image (right figures) mean the out of projection, shown in grayscale. 

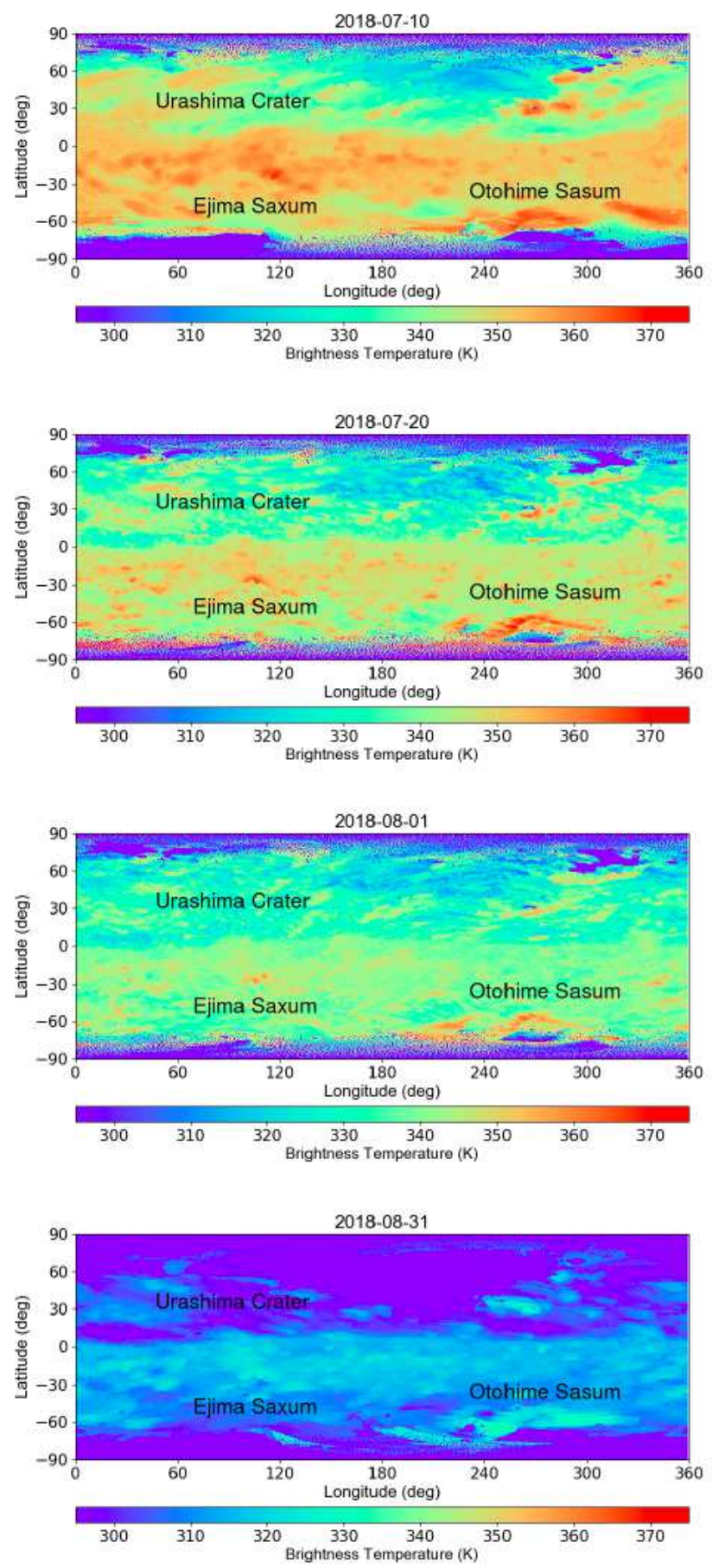

(a) Before correction
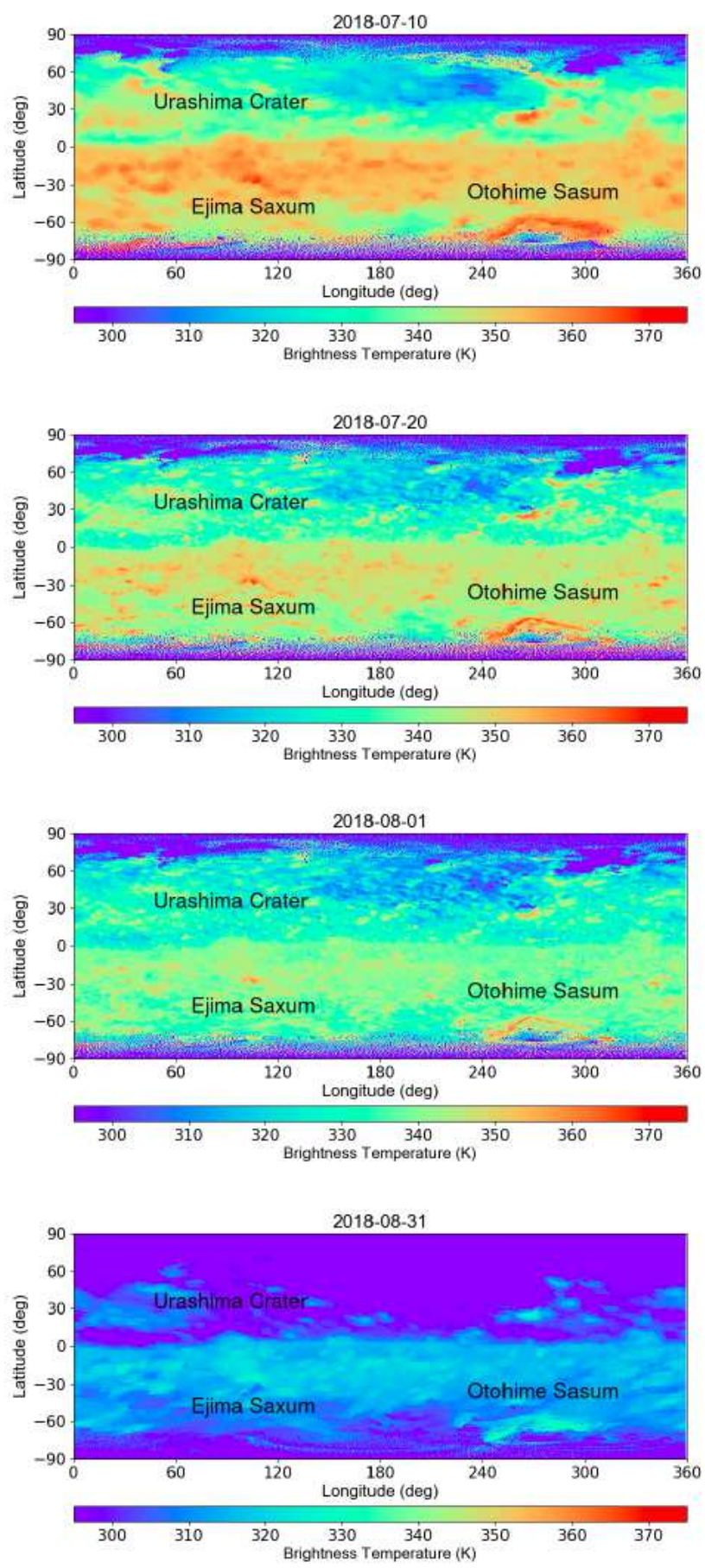

(b) After correction

\section{Figure 3}

Maximum temperature projected onto the geographic maps (Box-A, Box-C, Mid-Alt, and Box-B). (a) shows the before-correction map and (b) shows the after-correction map. These maps indicate that observed maximum temperatures merged and extracted combined all observed images for one rotation period of Ryugu projected onto the geographic map with $0.5^{\circ}$ by $0.5^{\circ}$ gridded. 

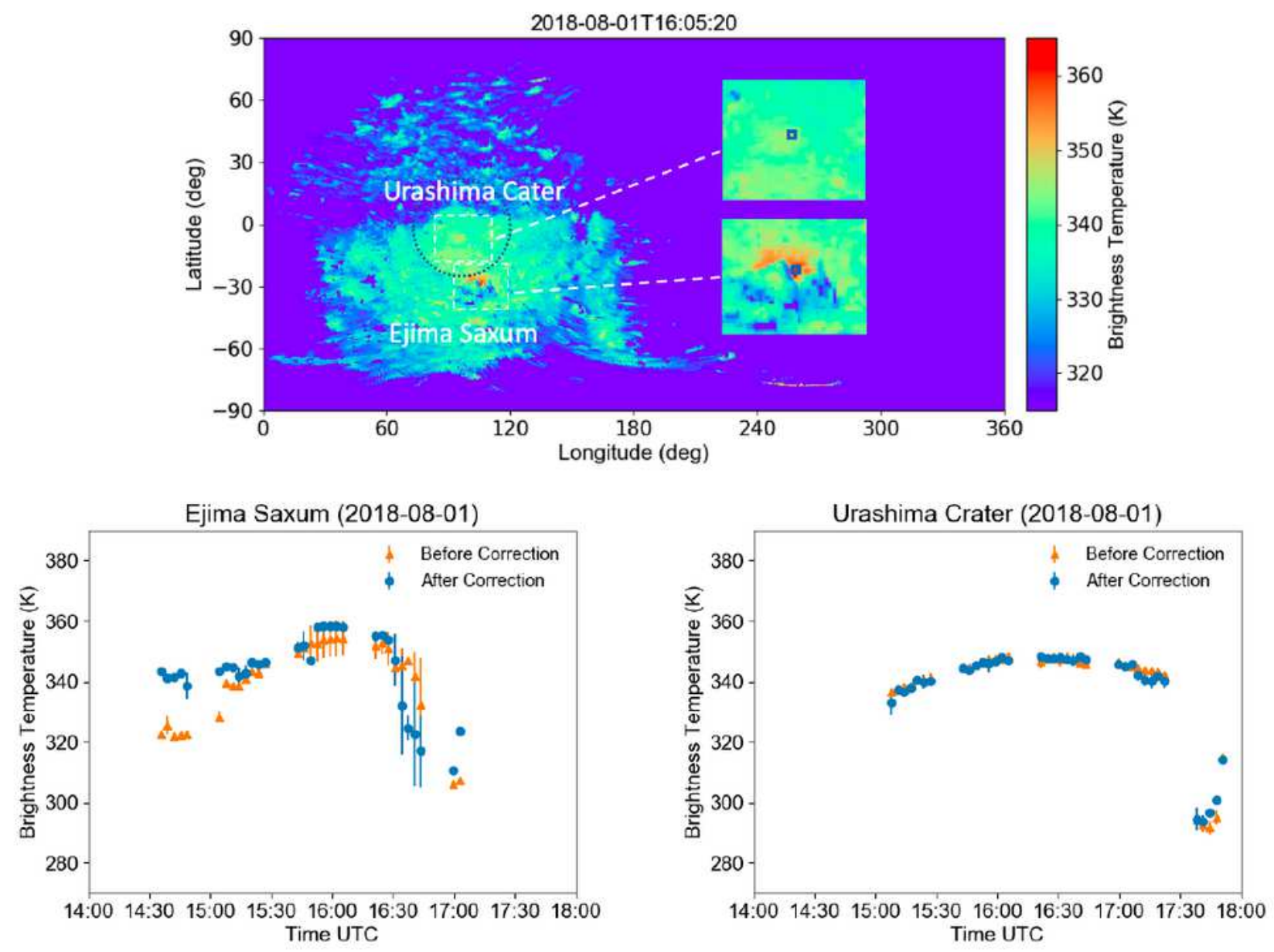

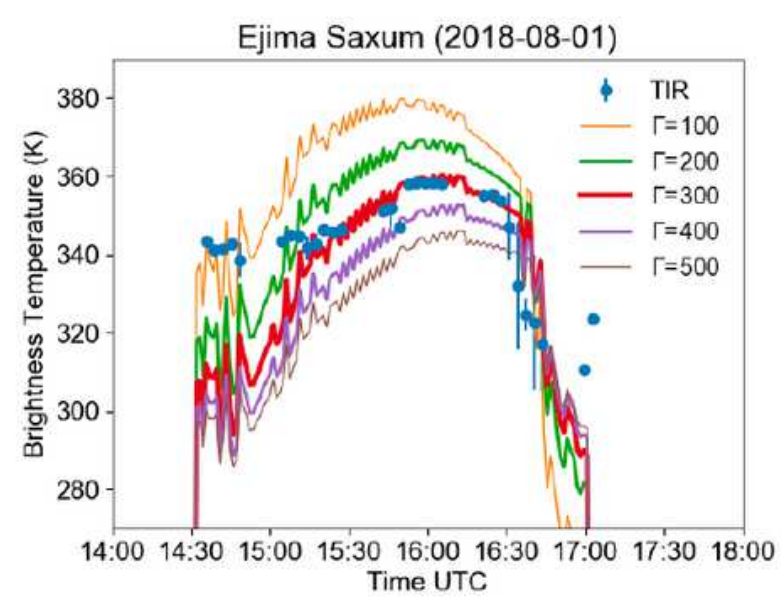

(a) Hot area of Ejima Saxum

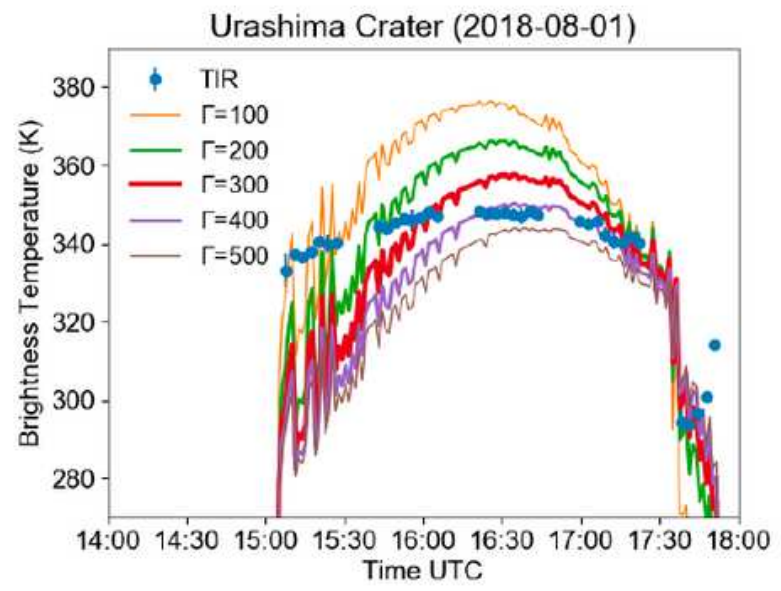

(b) Hot area of Urashima Crater

\section{Figure 4}

An example of one projection image (Mid-Alt16:05:20) using the shape model of Ryugu (SFM 800k v20180804) with $0.5^{\circ}$ by $0.5^{\circ}$ gridded (top) and the temperature profiles (middle and bottom). The temperature profiles were derived from 32 images extracted the hot areas of Ejima Saxum (a; Lat: $-28.0^{\circ}$, Lon: $106.0^{\circ}$ ) and Urashima crater (b; Lat: $-6.5^{\circ}$, Lon: $96.5^{\circ}$ ) as shown in the square area of the top image. These points indicate the observed brightness temperature of TIR by a time interval of 76 seconds, and 
the error bars are the observed temperature range of the minimum to maximum temperature. These orange points denote temperature before the geometric correction, and blue points denote temperature after geometric correction (middle). Comparison of the temperature profiles after the geometric correction with the simulated temperature profiles, TPM-1 (Takita et al. 2017), is shown (bottom). These lines indicate the simulated thermal inertia $\Gamma$ in steps of $100 \mathrm{Jm}-2 \mathrm{~s}-0.5 \mathrm{~K}-1$ using the shape model (SFM 200k v20180804).

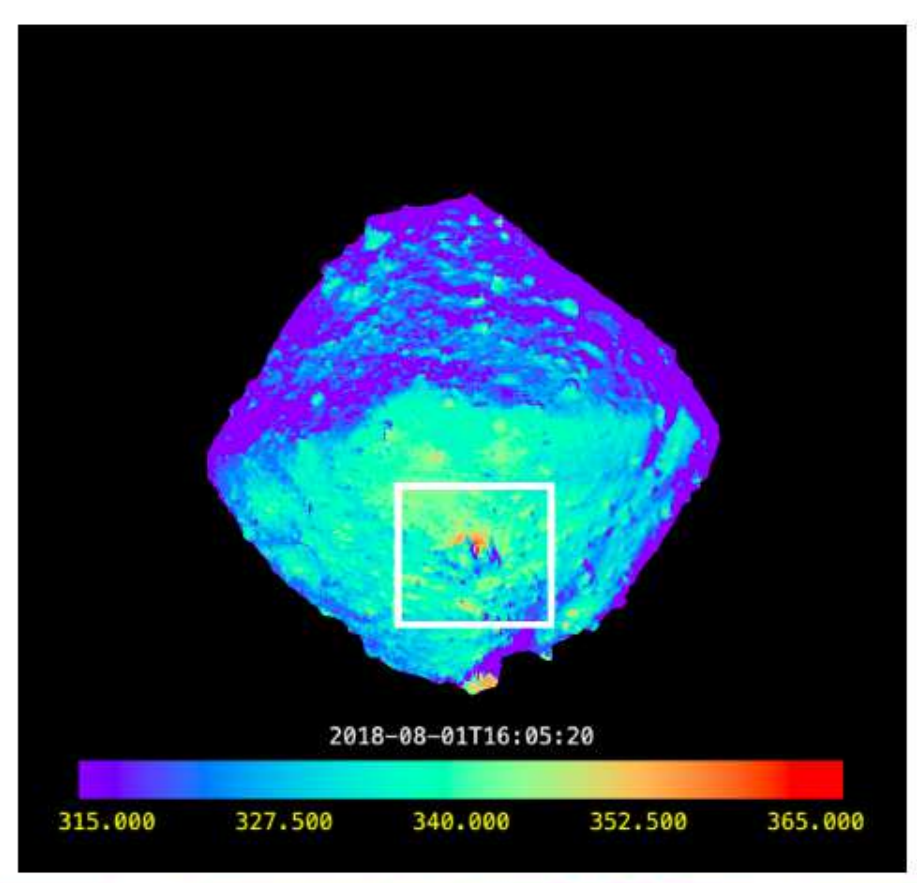

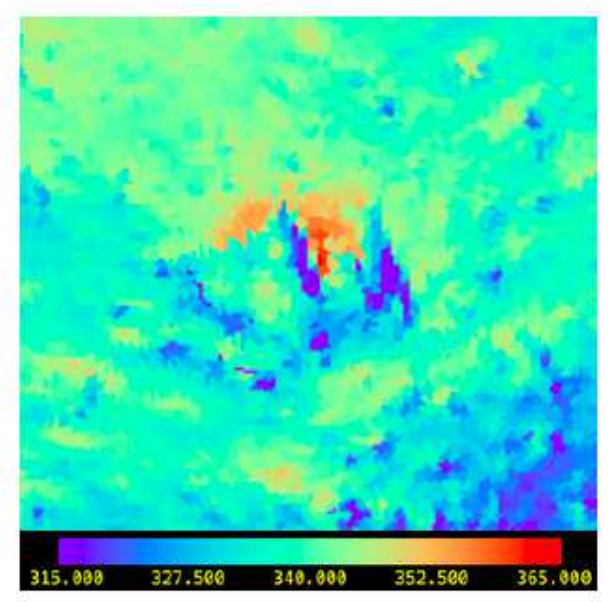

(a) Observed image

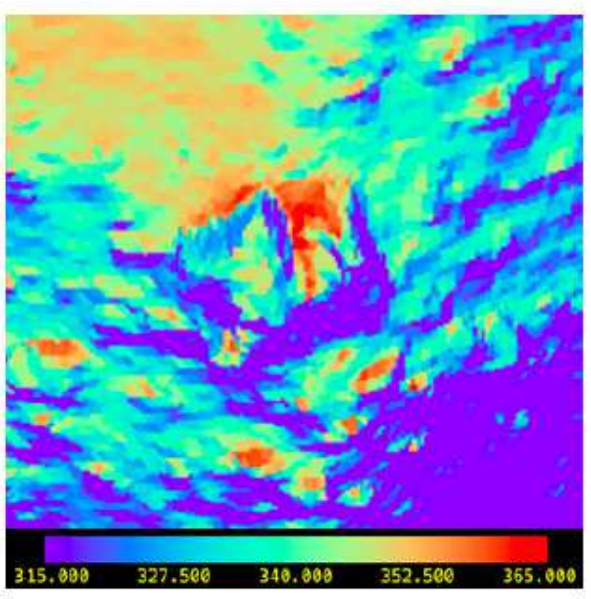

(b) Simulated image

(Scattering+Radiation)

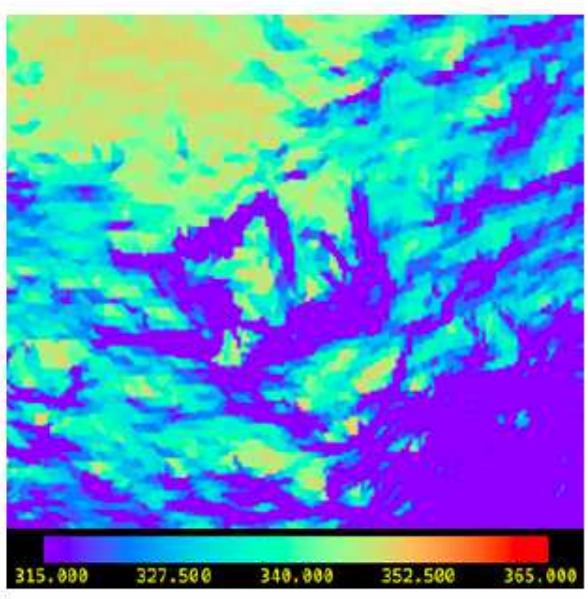

(c) Simulated image

(Scattering)

\section{Figure 5}

An example of the observed image (Mid-Alt 16:05:20) projected onto the shape model (SFM 200k v20180804) and comparison of the observed temperature and the numerically simulated temperature using TPM-1 (Takita et al. 2017). The top figure shows the projected temperature, and (a) shows the 
closed-up observed image of Ejima Saxum extracted as the square area of the top figure. (b) shows the simulated temperatures of Ejima Saxum, including the model of solar ray scatterings and secondary radiations between surrounding terrains. (c) shows the same figure of (b) except the effects of secondary radiations. The parameters of these simulations were assumed to be an emissivity of 1.0 , an albedo of 0.045 (Sugita et al. 2019), and a thermal inertia of $350 \mathrm{Jm}-2 \mathrm{~s}-0.5 \mathrm{~K}-1$.

\section{Supplementary Files}

This is a list of supplementary files associated with this preprint. Click to download.

- graphicalabstract.png 